\title{
Level of Melanotransferrin in Tissue and Sera Serves as a Prognostic Marker of Gastric Cancer
}

\author{
KOICHI SAWAKI ${ }^{1}$, MITSURO KANDA $^{1}$, SHINICHI UMEDA ${ }^{1}$, TAKASHI MIWA ${ }^{1}$, \\ CHIE TANAKA ${ }^{1}$, DAISUKE KOBAYASHI ${ }^{1}$, MASAMICHI HAYASHI ${ }^{1}$, SUGURU YAMADA ${ }^{1}$, \\ GORO NAKAYAMA ${ }^{1}$, KENJI OMAE ${ }^{2}$, MASAHIKO KOIKE ${ }^{1}$ and YASUHIRO KODERA ${ }^{1}$ \\ ${ }^{1}$ Department of Gastroenterological Surgery (Surgery II), \\ Nagoya University Graduate School of Medicine, Nagoya, Japan; \\ ${ }^{2}$ Department of Innovative Research and Education for Clinicians and Trainees (DiRECT), \\ Fukushima Medical University Hospital, Fukushima, Japan
}

\begin{abstract}
Aim: The aim of the study was to identify novel biomarkers that are vital for improving management of patients with gastric cancer (GC). Materials and Methods: An RNAsequencing analysis was conducted using gastric tissue from patients with metastatic GC. In vitro cell functions were evaluated by siRNA-mediated knockdown assays. A total of 230 pairs of gastric tissue were subjected to expression analysis of $m R N A$ and protein in situ. The serum levels of the candidate biomarker were determined by ELISA. Results: MELTF was identified as a candidate biomarker. Inhibition of MELTF expression suppressed the invasion ability of GC cells. Increased tissue MELTF mRNA expression was associated with shorter survival. Furthermore, staining intensity of tissue MELTF protein was linked to recurrence rates. Serum MELTF levels gradually were increased from healthy controls to advanced GC. Patients with high serum MELTF levels had poor prognosis. Conclusion: Both tissue and serum MELTF levels may serve as biomarkers of GC progression.
\end{abstract}

Despite important advances in multidisciplinary treatment, prognosis of patients with gastric cancer $(\mathrm{GC})$ remains poor $(1,2)$. Discovery of biomarkers for accurate staging, monitoring of recurrence and prognostication contribute to improvement of patient management.

Since GC is recognized as a heterogeneous disease both phenotypically and genetically, currently available biomarkers are inadequate (3). Particularly, biomarkers detectable in blood

Correspondence to: Mitsuro Kanda, Nagoya University Graduate School of Medicine, Department of Gastroenterological Surgery (Surgery II), 65 Tsurumai-cho, Showa-ku, Nagoya 466-8550, Japan. Tel: +81 527442249, Fax: +81 527442252, e-mail: m-kanda@med.nagoyau.ac.jp

Key Words: Gastric cancer, melanotransferrin, biomarker. samples are of practical value, because they can be measured repeatedly and non-invasively (4-7). However, conventional serum tumor markers, such as carbohydrate antigen (CA) 199, CA125 and carcinoembryonic antigen, may be widely used in clinical practice but are not ideal markers due to insufficient sensitivity and specificity in the risk stratification of advanced GC (8-10).

In this context, we sought to identify novel candidate genes involved in GC progression. Using an RNAsequencing analysis, we identified melanotransferrin (MELTF), which is a cell-surface glycoprotein (11). The aim of this study was to determine the oncological role of MELTF and significance of its expression in gastric tissue and sera as a biomarker of GC progression.

\section{Materials and Methods}

RNA-sequencing. To identify candidate genes associated with the metastatic potential of GC cells, surgically resected gastric tissue from four patients with distant metastasis of GC was subjected to RNA-sequencing analysis (HiSeq platform; Illumina, San Diego, CA, USA). We compared the expression levels of 57,749 genes in primary GC tissues with those of the corresponding normal gastric mucosa (12).

Cell lines and clinical samples. Fourteen GC cell lines (AGS, GCIY, IM95, KATOIII, MKN1, MKN7, MKN45, MKN74, N87, NUGC2, NUGC3, NUGC4, OCUM1 and SC-6-JCK) and a control, nonmalignant small intestinal epithelial cell line (FHs74) were obtained from the American Type Culture Collection (ATCC; Manassas, VA, USA) or the Japanese Collection of Research Bioresources Cell Bank (JCRB; Osaka, Japan). A total of 230 pairs of gastric tissue (tumor and the corresponding normal mucosa) were obtained from patients who underwent complete gastrectomy without neoadjuvant therapy between 2001 and 2014 as previously described (7). Since 2006, postoperative adjuvant therapy using S1 has been recommended for stage II or III GC patients unless contraindicated (13). As an external validation cohort, a public dataset comprising 1065 patients with GC was used $(14,15)$. 
Table I. List of genes overexpressed in primary cancerous tissues from patients with metastatic gastric cancer

\begin{tabular}{|c|c|c|c|c|c|c|}
\hline \multirow[t]{2}{*}{ Function } & \multirow[t]{2}{*}{ Symbol } & \multicolumn{2}{|c|}{ GC/Normal } & \multirow[b]{2}{*}{ Name } & \multicolumn{2}{|c|}{ Meta/GC } \\
\hline & & $\log _{2}$ & $p$-Value & & $\log _{2}$ & $p$-Value \\
\hline Cell-surface glycoprotein & $M E L T F$ & 3.27 & $<0.001$ & melanotransferrin & -0.19 & 0.738 \\
\hline \multirow{3}{*}{ Trafficking protein } & DNAJC12 & 4.15 & $<0.001$ & DNAj heat shock protein family member $\mathrm{C} 12$ & -1.16 & 0.104 \\
\hline & $R B P 4$ & 4.25 & $<0.001$ & Retinol binding protein 4 & 1.51 & 0.052 \\
\hline & SYT7 & 4.29 & $<0.001$ & Synaptotagmin 7 & 0.30 & 0.628 \\
\hline \multirow[t]{4}{*}{ Transcription factor } & FNDC1 & 4.50 & $<0.001$ & Fibronectin type III domain containing 1 & -0.89 & 0.159 \\
\hline & GNG4 & 4.84 & $<0.001$ & G protein subunit gamma 4 & 0.29 & 0.730 \\
\hline & ELF5 & 5.00 & $<0.001$ & E74 like ETS transcription factor 5 & -0.85 & 0.332 \\
\hline & HOXC10 & 6.49 & $<0.001$ & Homeobox C10 & 1.68 & 0.075 \\
\hline \multirow[t]{3}{*}{ Cell membrane receptor } & GRB7 & 3.98 & $<0.001$ & Growth factor receptor bound protein 7 & -0.03 & 0.972 \\
\hline & $U T S 2 R$ & 4.50 & $<0.001$ & Urotensin 2 receptor & 0.50 & 0.568 \\
\hline & TNFRSF11B & 4.57 & $<0.001$ & TNF receptor superfamily member $11 \mathrm{~b}$ & 0.53 & 0.427 \\
\hline \multirow[t]{4}{*}{ Cellular adhesin } & COMP & 3.15 & $<0.001$ & Cartilage oligomeric matrix protein & 0.91 & 0.107 \\
\hline & CLDN1 & 3.27 & $<0.001$ & Claudin 1 & 0.71 & 0.157 \\
\hline & THBS2 & 3.76 & $<0.001$ & Thrombospondin 2 & 0.20 & 0.776 \\
\hline & THBS4 & 4.01 & $<0.001$ & Thrombospondin 4 & 0.95 & 0.279 \\
\hline Growth factor & INHBA & 3.76 & $<0.001$ & Inhibin beta A subunit & -0.37 & 0.503 \\
\hline \multirow{3}{*}{ Mediator of neural transmission } & $V S N L 1$ & 4.04 & $<0.001$ & Visinin like 1 & 1.09 & 0.153 \\
\hline & $C P L X 2$ & 4.36 & $<0.001$ & Complexin 2 & 1.88 & 0.244 \\
\hline & $N P Y$ & 4.86 & $<0.001$ & Neuropeptide $Y$ & 0.09 & 0.901 \\
\hline \multirow[t]{4}{*}{ Metabolic enzyme } & PADI2 & 3.01 & $<0.001$ & Peptidyl arginine deiminase 2 & -1.29 & 0.076 \\
\hline & KLK10 & 3.26 & $<0.001$ & Kallikrein related peptidase 10 & -0.76 & 0.298 \\
\hline & AKRlC4 & 3.28 & $<0.001$ & Aldo-keto reductase family 1 member $\mathrm{C} 4$ & 0.59 & 0.406 \\
\hline & $P L A 2 G 2 A$ & 3.70 & $<0.001$ & Phospholipase A2 group IIA & -0.43 & 0.453 \\
\hline \multirow[t]{2}{*}{ Regulator of cell cycle } & $C D C 25 B$ & 3.17 & $<0.001$ & Cell division cycle $25 \mathrm{~B}$ & -0.66 & 0.395 \\
\hline & CCNE1 & 3.41 & $<0.001$ & Cyclin E1 & -1.06 & 0.071 \\
\hline
\end{tabular}

GC, Primary gastric cancer tissue; Normal, corresponding adjacent normal gastric tissue; Meta, hepatic metastasis tissue.

Transcription of MELTF and PCR array analysis. The levels of MELTF mRNA were analyzed using a real-time quantitative reverse-transcription PCR (RT-PCR) analysis. As a reference gene, glyceraldehyde-3-phosphate dehydrogenase $(G A P D H)$ was used (16). To investigate the involvement of MELTF by identifying cancer-related genes expressed simultaneously with MELTF, PCR array analysis was performed using the human epithelial to mesenchymal transition (EMT) RT2 Profiler PCR Array (Qiagen, Hilden, Germany) (17).

Knockdown of MELTF expression. MELTF was silenced by transfection with small interfering RNA (siRNA) as described previously using LipoTrust EX Oligo (Hokkaido System Science, Sapporo, Japan) (17). To confirm MELTF knockdown, both mRNA and protein expression of MELTF were analyzed. A rabbit polyclonal antibody against MELTF (NBP1-85777PEP; Novus Biologicals, Centennial, CO, USA; dilution 1:1000) was used for western blot analysis (18).

Cell functional analyses. Cell proliferation was evaluated with the Cell Counting Kit-8 (Dojindo Molecular Technologies, Inc., Kumamoto, Japan) $(19,20)$. We determined the invasive ability of GC cells using BioCoat Matrigel invasion chambers (BD Biosciences, Bedford, MA, USA). Wound-healing assay was performed to evaluate cell migration (17). The CytoSelect 48-Well
Cell Adhesion Assay (Cell Biolabs, Inc., San Diego, CA) was employed to analyze adherence of GC cells to the extracellular matrix components (18).

Immunohistochemistry (IHC). In situ MELTF protein expression was determined with IHC in 84 representative patients who had well-preserved sections of GC tissues. Formalin-fixed paraffinembedded sections were incubated for $1 \mathrm{~h}$ at room temperature with a rabbit polyclonal antibody against MELTF (NBP1-85777PEP, Novus Biologicals) diluted 1:100. We graded the patients according to the staining intensity of MELTF in GC component: none, weak, moderate, and strong staining as previously described (21).

Enzyme-linked immunosorbent assay (ELISA). The serum levels of MELTF were determined in 20 healthy controls and 68 patients with GC. Adults ( $n=20$, median age 42 years) in the healthy controls had annual medical checkups (chest X-rays, blood tests and physical examination) and were not under disease treatment. All patients were diagnosed as adenocarcinoma of the stomach by upper gastrointestinal endoscopy before blood collection for the ELISA assay. We collected blood samples within 7 days before gastrectomy. Serum was immediately separated and stored at $-80^{\circ} \mathrm{C}$. MELTF ELISA Kit (CSB-EL013754HU, CUSABIO, Wuhan, PR China) was used and all samples were assayed in duplicate (22). 
Statistical analyses. Differences in the data between the two groups were analyzed by the Mann-Whitney test or $\chi^{2}$ test. The receiveroperating characteristic (ROC) curve was used to assess goodnessof-fit by calculating the area under the curve (AUC). Survival data was analyzed with Kaplan-Meier curves. To identify prognostic factors, multivariable regression analysis was performed using the Cox proportional hazards model, and differences between variables with $p<0.05$ were entered in the final model. JMP 13 software (SAS Institute Inc., Cary, NC, USA) was used for all statistical analyses. A $p$-value of less than 0.05 was considered to indicate statistically significant differences.

\section{Results}

Detection of MELTF. As a result of an RNA-sequencing analysis, 25 candidates identified that were highly expressed in GC tissues compared to the noncancerous tissue and comparably expressed in primary and metastatic tissue (Table I). Of them, we selected MELTF for further investigation, because it encodes for a cell-surface glycoprotein that could be released into the circulatory system, and no previous reports were found regarding GC.

Expression in GC cell lines. The MELTF mRNA levels were fivefold higher in MKN7, NUGC4, OCUM1, AGS, MKN1, and N87 cells than in FHs74 cells. No clear trends in MELTF mRNA levels were indicated based on the differentiation of the GC cell lines. Three epithelial mesenchymal transitionrelated genes encoding vacuolar protein sorting 13 homolog A (VPS13A), snail family transcriptional repressor 3 (SNAI3), and Erb-B2 receptor tyrosine kinase 3 (ERBB3) were expressed at levels with correlation coefficient $>0.6$ compared with MELTF (Figure 1a).

Effect of MELTF knockdown on biological activities of gastric cancer cells. N87, AGS, and MKN1 were differentiated-type cells displaying high levels of MELTF mRNA expression. NUGC4 and OCUM1 were undifferentiated-type cells also displaying high MELTF mRNA expression. Based on the characteristics of the cells (e.g. invasion and migration abilities), we selected MKN1 and AGS from the differentiatedtype cells, and NUGC4 from the undifferentiated-type cells for the knockdown experiments. To evaluate the oncological functions of MELTF in GC cells, MELTF was silenced in MKN1, AGS, and NUGC4 cells by specific siRNAs. After confirming knockdown efficiency of mRNA and protein expression (Figure 1b), we compared cell proliferation, invasiveness, migration, and adhesion of MKN1 and AGS cells with and without MELTF knockdown. Inhibition of MELTF expression significantly decreased the proliferation of MKN1 cells (Figure 1c) and invasion of MKN1 and AGS cells (Figure 2a). Furthermore, the migration of AGS cells was reduced by siMELTF (Figure 2b). However, the silencing of MELTF had little effect on the adhesive ability (Figure 2c).
Prognostic value of tissue MELTF mRNA expression. There were 165 males and 65 females aged 26 to 96 years $[66.3 \pm 10.8$ years, mean \pm standard deviation (SD)]. With respect to pathological stage, 50,71, and 109 patients were in stages I, II, and III, respectively, indicating that all 230 patients underwent curative gastrectomy. GC tissue samples had significantly higher mean levels of MELTF mRNA than the adjacent normal tissues. The AUC value of MELTF levels was 0.749 for detection of cancer-related death within 2 years after gastrectomy, and the optimal cutoff value was 0.00248 (sensitivity $91 \%$, specificity $55 \%$ ). Patients were classified into the low MELTF (below the cut-off value) or high MELTF (equal to or above the cut-off value) groups. The high MELTF group had a significantly lower overall survival rate than the low MELTF group (5-year survival rates, $78 \%$ and $89 \%$, respectively; Figure 3a). Furthermore, the disease-free survival rate was significantly lower in the high MELTF group than in the low MELTF group (3-year disease-free survival rates, $64 \%$ and $89 \%$, respectively, $p<0.001)$. The frequencies of peritoneal and lymph node recurrences were significantly higher in the high MELTF group than in the low MELTF group, whereas there was no significant difference in that of hematogenous recurrences. Consistent results were obtained in the analysis of the extra-validation cohort (Figure 3b).

Next, we investigated the correlation between MELTF expression and clinicopathological characteristics in patients. High MELTF expression in GC tissue was significantly correlated with differentiated tumor and vascular invasion. High MELTF mRNA expression in GC tissue was found to be a significant prognostic factor together with CA19-9 > 37 IU/ml, larger tumor size, pT4, invasive growth, lymphatic involvement, vascular invasion and nodal metastasis in the univariate analysis of overall survival. Multivariate analysis identified lymph nodal metastasis and high MELTF mRNA levels as independent prognostic factors for overall survival (Table II).

Expression of MELTF protein in situ using immunohistochemistry. Examples of each staining intensity are shown in Figure 3a. Forty-three, 20, 18, and 3 patients were categorized as none, weak, moderate, and strong staining, respectively. Prevalence of recurrence after surgery gradually increased as the intensity of staining of MELTF increased (Figure 3c).

Prognostic value of serum MELTF levels. Fifty-one males and 17 females were included for the serum analysis and they were aged 24 to 88 years $(65.1 \pm 12.6$ years, mean \pm SD). Based on the UICC TNM Classification of Malignant Tumors, 8 th edition, 17, 15, 16, and 20 patients were in stage I, II, III, and IV, respectively. Twenty-seven and 41 patients were diagnosed with differentiated and undifferentiated GC. Serum MELTF levels of the 20 healthy controls ranged from 0 to $105.2 \mathrm{pg} / \mathrm{ml}(15.9 \pm 28.0 \mathrm{pg} / \mathrm{ml}$, mean \pm SD). Patients with GC $(n=68)$ had significantly higher preoperative serum 
$\mathbf{a}$
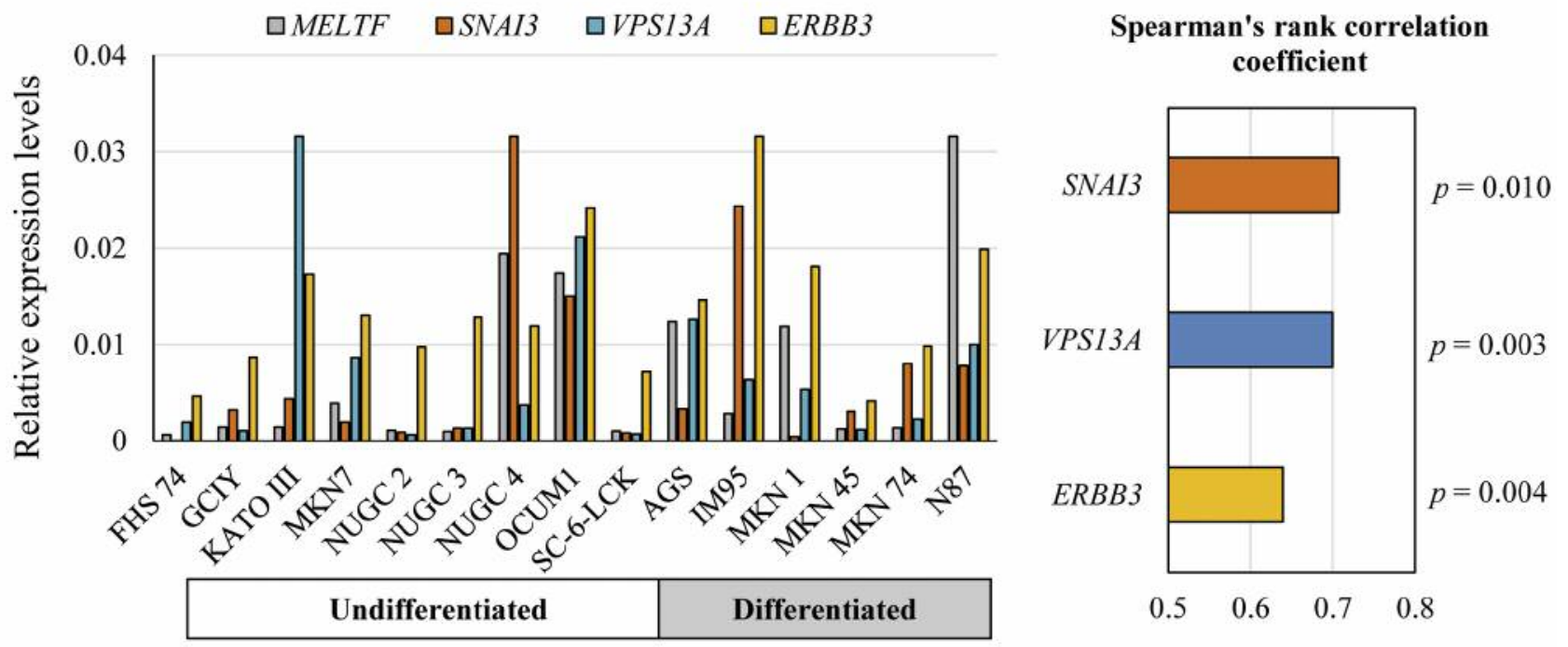

b
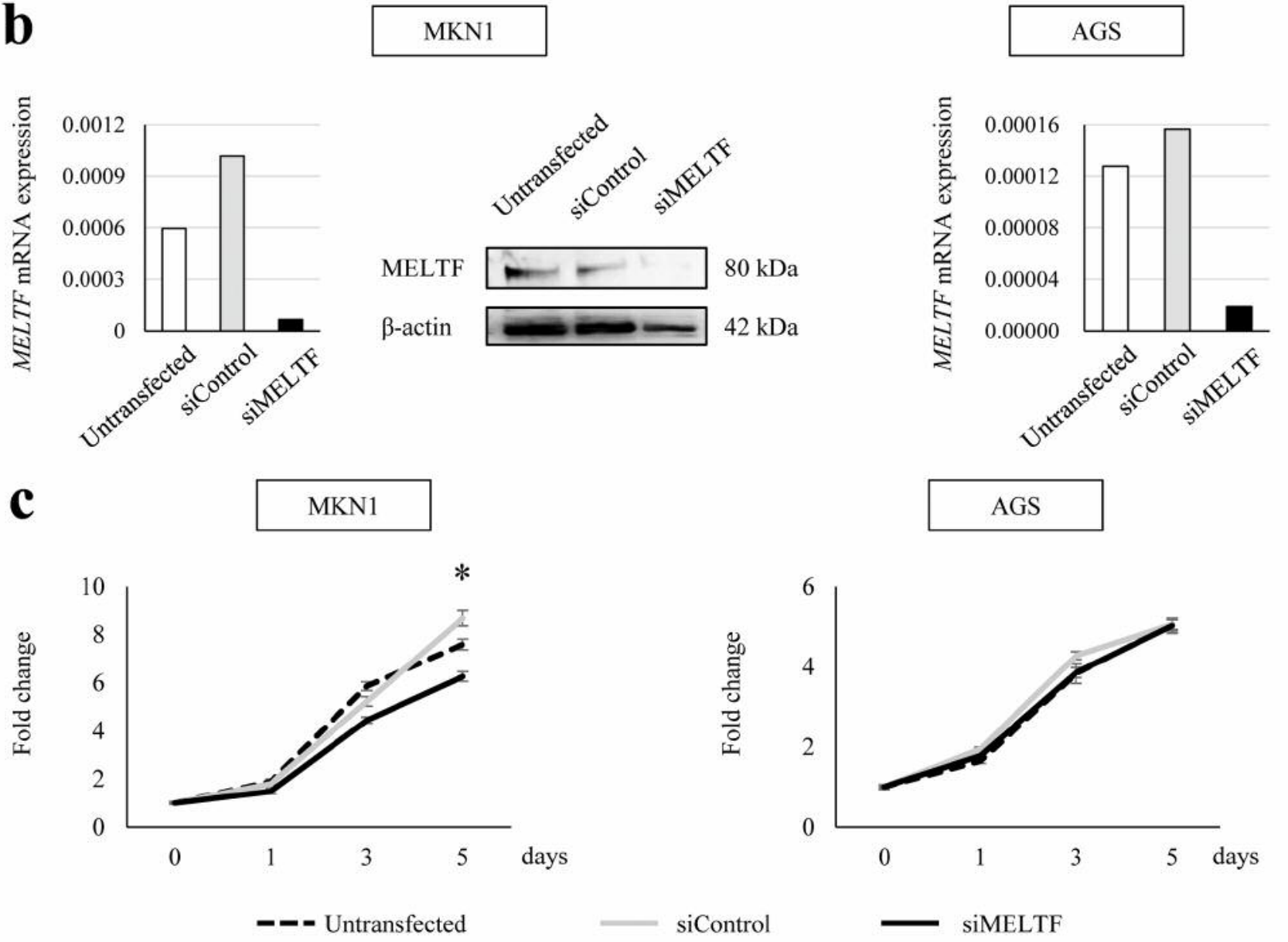

Figure 1. Expression analysis of cell lines and effects of siRNA-mediated knockdown of MELTF expression. (a) Expression of MELTF and genes at similar differential levels were identified using polymerase chain reaction (PCR) array analysis. MELTF mRNA levels positively correlated with those of SNAI3, VPS13A, and ERBB3. (b) Silencing of MELTF in MKN1 and AGS cells was examined using PCR and western blot analysis. (c) Cell proliferation of MKN1 and AGS cells transfected with siMELTF, control siRNA, or untreated. $* p<0.05$. Error bars indicate standard deviation. siRNA, Small interfering RNA. 
Table II. Prognostic factors for overall survival in 230 patients with gastric cancer.

\begin{tabular}{|c|c|c|c|c|c|c|c|}
\hline \multirow[t]{2}{*}{ Variables } & \multirow[t]{2}{*}{$\mathrm{n}$} & \multicolumn{3}{|c|}{ Univariate } & \multicolumn{3}{|c|}{ Multivariate } \\
\hline & & HR & $95 \% \mathrm{CI}$ & $p$-Value & HR & $95 \% \mathrm{CI}$ & $p$-Value \\
\hline Age $(\geq 65)$ & 131 & 0.96 & $0.53-1.85$ & 0.896 & & & \\
\hline Gender (female) & 65 & 1.1 & $0.54-2.12$ & 0.774 & & & \\
\hline CEA $(>5 \mathrm{ng} / \mathrm{ml})$ & 35 & 1.39 & $0.60-2.86$ & 0.42 & & & \\
\hline CA19-9 (37>IU/ml) & 39 & 2.47 & $1.17-4.84$ & 0.019 & 1.56 & $0.72-3.15$ & 0.245 \\
\hline Tumor location (lower third) & 81 & 0.77 & $0.38-1.45$ & 0.421 & & & \\
\hline Tumor size $(\geq 50 \mathrm{~mm})$ & 106 & 3.06 & $1.60-6.23$ & 0.001 & 1.79 & $0.92-3.72$ & 0.087 \\
\hline Macroscopic type (type 4/5) & 22 & 2.01 & $0.82-4.28$ & 0.121 & & & \\
\hline Tumor depth (pT4, UICC) & 83 & 3.65 & $1.95-7.07$ & $<0.001$ & 1.87 & $0.94-3.87$ & 0.076 \\
\hline Differentiation (undifferentiated) & 131 & 1.86 & $0.98-3.72$ & 0.059 & & & \\
\hline Lymphatic involvement & 193 & 5.4 & $1.65-33.3$ & 0.003 & 0.96 & $0.20-6.97$ & 0.958 \\
\hline Vascular invasion & 143 & 3.81 & $1.84-8.89$ & $<0.001$ & 1.19 & $0.49-3.25$ & 0.715 \\
\hline Invasive growth & 71 & 2.21 & $1.17-4.11$ & 0.015 & 1.6 & $0.80-3.16$ & 0.181 \\
\hline Lymph node metastasis & 143 & 12.6 & $4.49-52.4$ & $<0.001$ & 9.48 & $2.78-46.2$ & $<0.001$ \\
\hline Adjuvant chemotherapy & 107 & 1.43 & $0.77-2.67$ & 0.254 & & & \\
\hline High $M E L T F$ & 137 & 3 & $1.54-6.28$ & 0.001 & 3.66 & $1.76-8.21$ & $<0.001$ \\
\hline
\end{tabular}

CI, Confidence interval; UICC, Union for International Cancer Control.

MELTF levels $(388 \pm 1834 \mathrm{pg} / \mathrm{ml}$, mean \pm SD) than the healthy controls. Serum MELTF levels gradually increased along with disease stages (Figure 3d). We set the cut-off of serum MELTF levels at $105.2 \mathrm{pg} / \mathrm{ml}$, which were the highest levels in the healthy controls. The sensitivity, specificity, false positive, and false negative at the cutoff value of MELTF $105.2 \mathrm{pg} / \mathrm{ml}$ were $50 \%, 100 \%, 0 \%$ and $50 \%$, respectively. Using this cut-off, GC patients were classified into the MELTF-positive (equal to or above the cut-off) and MELTFnegative (less than the cut-off) groups. Patients in the MELTF-positive group had significantly shorter survival than those in the MELTF-negative group (Figure 3e).

\section{Discussion}

RNA-sequencing analysis using tissue from patients with metastatic GC, identified MELTF as a candidate biomarker. Expression levels of MELTF were correlated with those of three EMT-related genes, SNAI3, VPS13A, and ERBB3, and knockdown of MELTF expression attenuated proliferation, invasion, and migration abilities of GC cells. Tissue and serum MELTF levels were associated with disease recurrence and prognosis.

MELTF is a cell-surface glycoprotein initially found on melanoma cells, and it belongs to the transferrin superfamily $(11,23)$. MELTF is related to transferrin in tertiary structure and has the physiological role of iron cellular uptake $(11,24$, 25). In malignancy, it has been reported that MELTF is significantly overexpressed in melanoma and associated with increased proliferation of melanoma cells $(11,25,26)$. Additionally, recent reports have confirmed MELTF as a potential serological marker for colorectal cancer $(27,28)$. However, the role of MELTF in GC is poorly understood.

PCR array analysis showed that SNAI3, VPS13A, and ERBB3 were expressed together with MELTF with high correlation coefficient. SNAI3 plays a role in mesodermal formation during embryogenesis and acts as a transcriptional repressor implicated in the EMT process $(29,30)$. VPS13A is reported to control the process of cycling through the trans-Golgi network and the plasma membrane, lysosomes and endosomes $(16,31)$. It has been reported that VPS13A is expressed in various cancer cells (32). ERBB3 is a member of the epidermal growth factor receptor (EGFR) family and has been reported that this gene is overexpressed in GC (33-35). We showed here that MELTF contributes to the proliferation, invasion, and migration ability of GC cells. Our present findings support the conclusion that MELTF acts as a putative oncogene in GC.

In our cohort, high expression of tissue MELTF mRNA was significantly associated with shorter disease-free and overall survival after curative resection. These findings are consistent with the findings from our analysis of a validation cohort (14). Multivariate analysis showed that high mRNA levels of MELTF are an independent prognostic factor for survival. Moreover, expression status of tissue MELTF can be evaluated by immunohistochemical staining. These results highlighted the value of MELTF expression as a biomarker for GC progression.

Next, we detected MELTF in serum samples, because blood sampling is less invasive and readily accessible $(6,36)$. MELTF levels in serum samples of GC patients were significantly higher than that in healthy controls. Because preoperative serum MELTF levels gradually increased along with higher UICC 


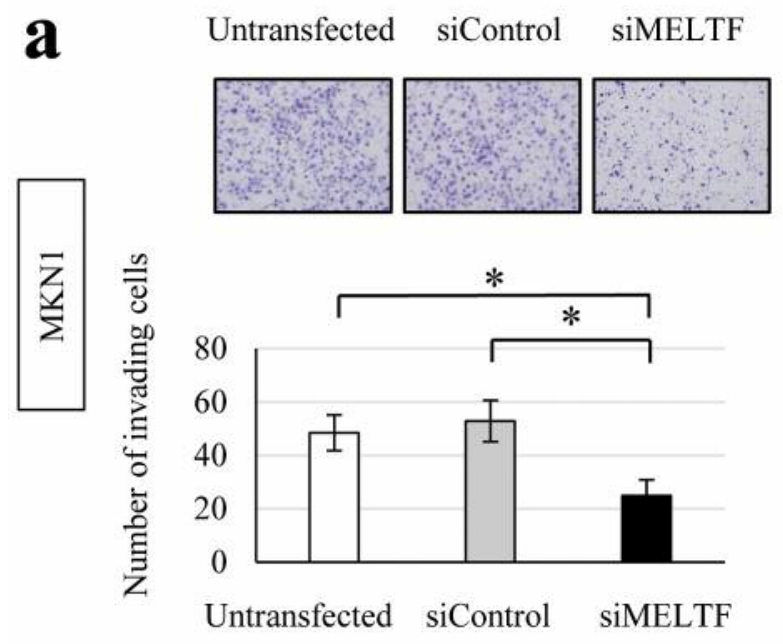

Untransfected siControl siMELTF
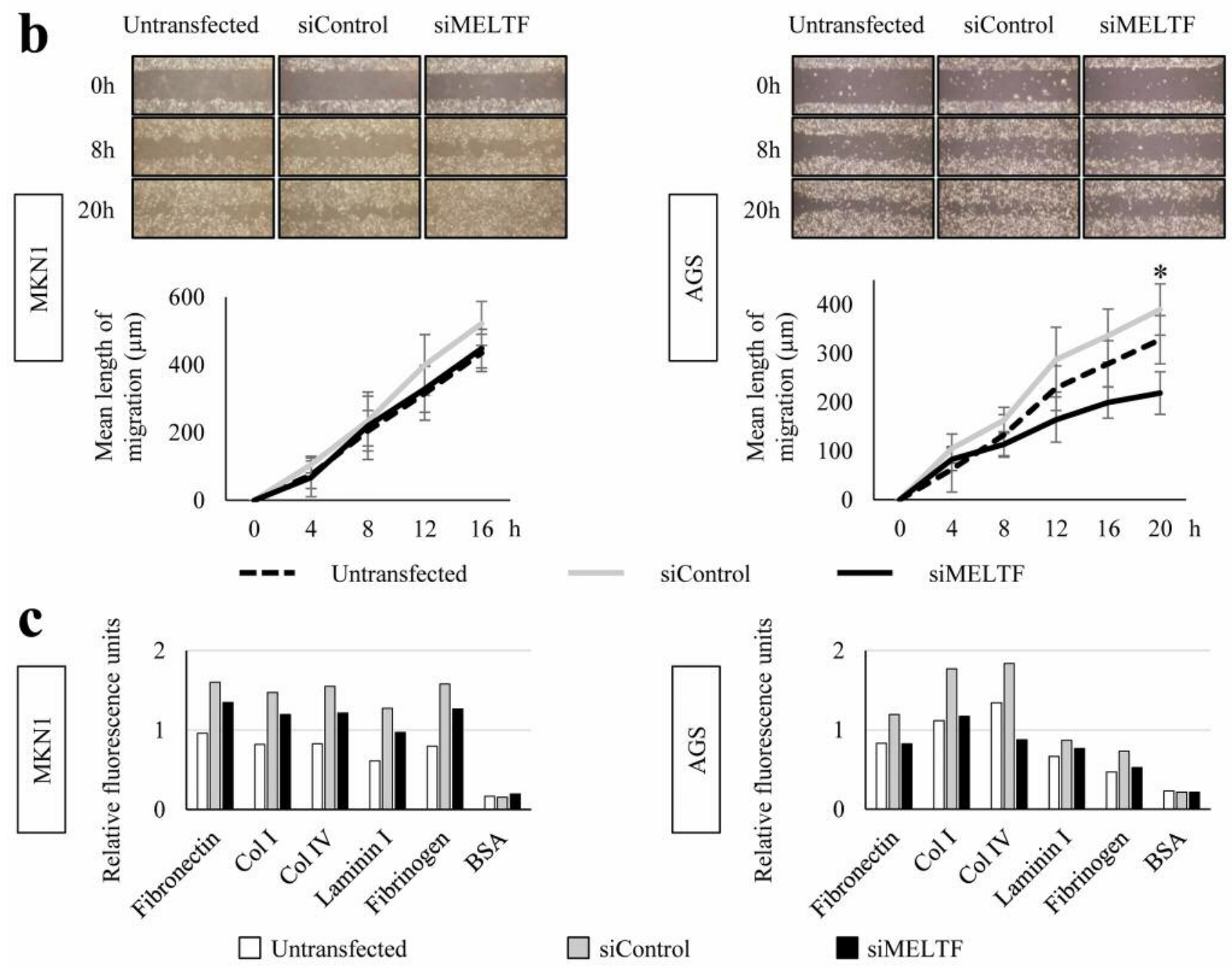

Figure 2. Effect of inhibition of MELTF on MKN1 and AGS cells. (a) Cell invasion assay of MKN1 and AGS cells transfected with siMELTF, control siRNA, or untreated (200x magnification). (b) Migration assays in MKN1 and AGS cells transfected as indicated. Graph shows mean length of migration at the indicated times. (c) Cell adhesion assay in MKN1 and AGS cells transfected as indicated. *p<0.05. Error bars indicate standard deviation. siRNA, Small interfering RNA. 
a

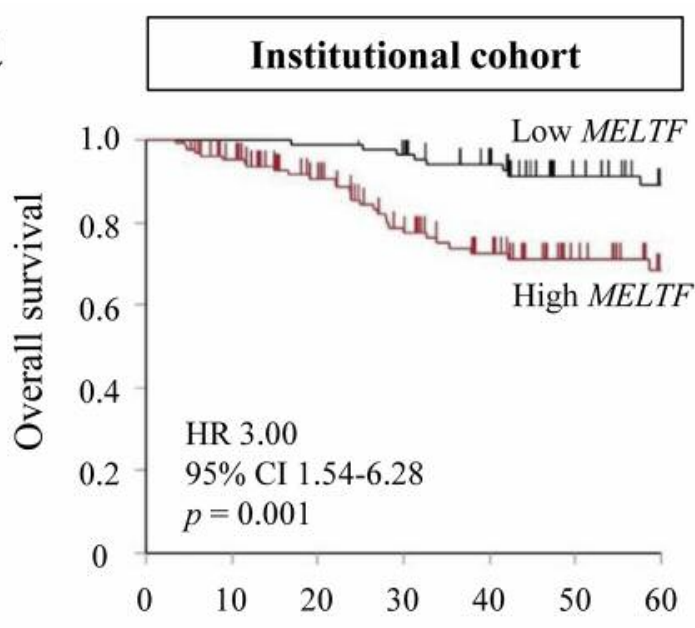

No. at risk

Months after surgery

$\begin{array}{llllllll}\text { Low MELTF } & 137 & 113 & 92 & 70 & 55 & 36 & 25\end{array}$

$\begin{array}{llllllll}\text { High MELTF } & 93 & 91 & 87 & 82 & 70 & 53 & 42\end{array}$

c

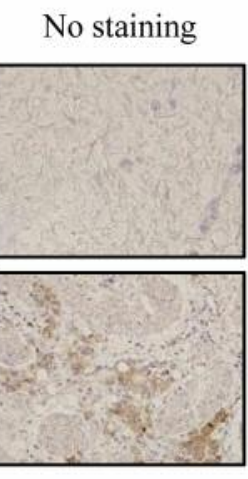

Moderate
Weak
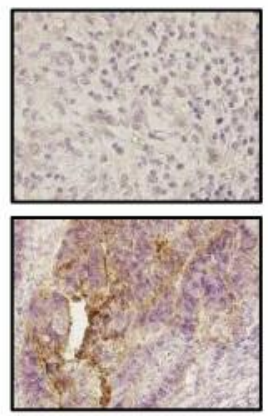

Strong d

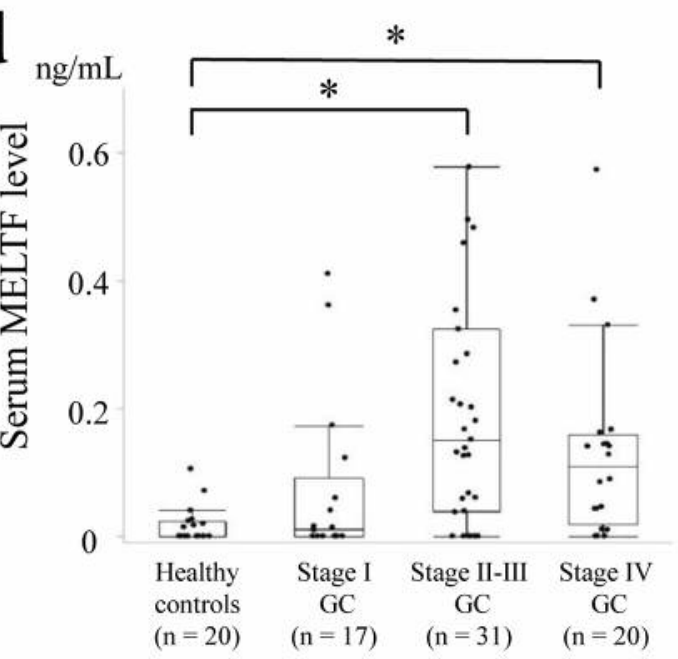

b

Extra-validation cohort

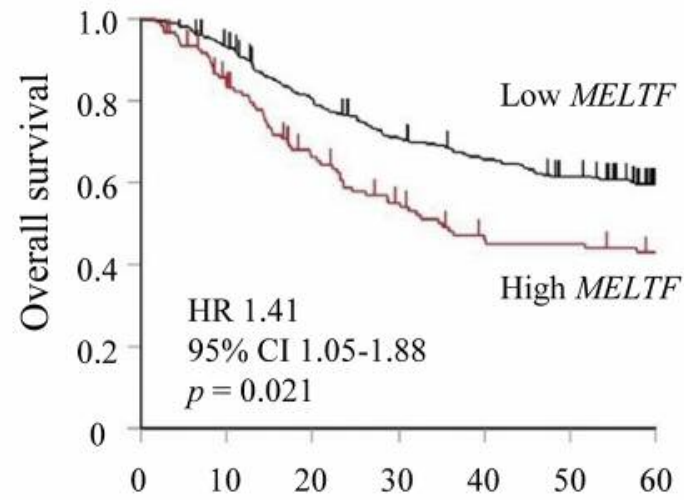

No. at risk

Months after surgery

$\begin{array}{llllllll}\text { Low MELTF } & 227 & 202 & 170 & 149 & 136 & 125 & 111\end{array}$ $\begin{array}{llllllll}\text { High MELTF } & 217 & 180 & 143 & 119 & 101 & 95 & 77\end{array}$

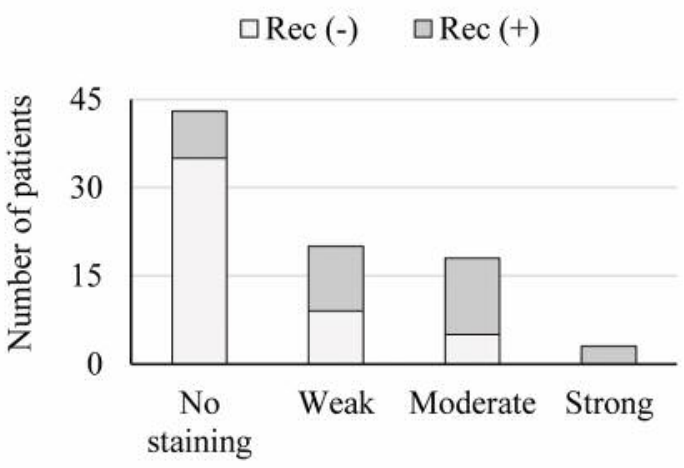

$\mathbf{e}$

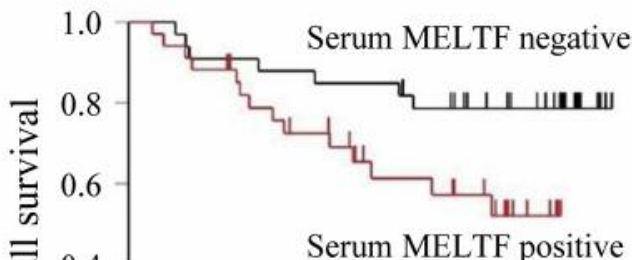

HR 2.52

$0.2-95 \%$ CI $1.04-6.67$ $p=0.040$

0

$\begin{array}{llllll}0 & 10 & 20 & 30 & 40 & 50\end{array}$

No. at risk

Months after surgery

$\begin{array}{lllllll}\text { MELTF negative } & 34 & 31 & 30 & 29 & 21 & 7\end{array}$

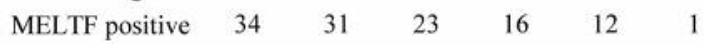

Figure 3. Overall survival of patients with stage I-III gastric cancer according to tissue mRNA levels of MELTF in our institutional cohort (a) and the extra-validation cohort (b). (c) Immunohistochemical staining. Representative data showing none, weak, moderate, and strong staining for MELTF expression (400x magnification). The results show a positive correlation between the staining intensity of MELTF in gastric cancer component and frequency of recurrence. (d) Measurement of serum MELTF levels. Stepwise elevation of serum MELTF levels in healthy controls and patients with gastric cancer. ${ }^{*} p<0.05$. Error bars indicate standard deviation. (e) Patients in the MELTF-positive group had poorer prognosis compared with those in the MELTF-negative group. 
stage and were associated with poor overall survival, examining preoperative MELTF serum levels may help predict patient prognosis. Shin et al. have conducted quantitative proteomic analysis to identify serum biomarkers for colorectal cancer and confirmed MELTF as a potential serological marker (27). They measured levels of MELTF by ELISA in 325 samples from 77 healthy controls and 228 patients with colorectal cancer and found that serum MELTF levels were elevated in patients with colorectal cancer (27). Detection of MELTF in serum samples is an ideal method for screening and emphasizes the clinical practicality of evaluating MELTF expression.

Several limitations of this study should be acknowledged. Further pathway analysis is desirable to understand the oncological roles of MELTF in GC. The relatively small sample size limited the statistical power. Unfortunately, very limited cases experienced disease recurrences after measurement of serum MELTF levels. Therefore, it is difficult to analyze correlations between serum MELTF levels and recurrent patterns this time.

To further evaluate the diagnostic performance of serum MELTF levels, a prospective study to determine if serum MELTF levels normalize after resection of primary gastric cancer and rise prior to radiographic evidence of disease recurrences is needed.

In conclusion, MELTF acts as a putative oncogene in GC and its levels in primary GC tissues and serum samples may be a potential biomarker.

\section{Conflicts of Interest}

The Authors have no conflicts of interest directly relevant to the content of this article.

\section{Authors' Contributions}

KS and MK conceived the study concept and design, analyzed data and wrote the manuscript. MK, SU, TM, CT, DK, MH, SY, GN, $\mathrm{KO}, \mathrm{MK}$ and $\mathrm{YK}$ contributed to data acquisition and interpretation. KO contributed to statistical analysis. CT, DK, MH, SY, GN, MK, YK and KO revised the draft. All authors have read and approved the final version of the manuscript.

\section{Acknowledgements}

The Authors thank Edanz Group (www.edanzediting.com/ac) for editing a draft of this article.

\section{References}

1 Kanda M, Tanaka C, Kobayashi D, Mizuno A, Tanaka Y, Takami H, Iwata N, Hayashi M, Niwa Y, Yamada S, Fujii T, Sugimoto H, Murotani K, Fujiwara M and Kodera Y: Proposal of the coagulation score as a predictor for short-term and long-term outcomes of patients with resectable gastric cancer. Ann Surg Oncol 24(2): 502-509, 2017. PMID: 27600621. DOI: 10.1245/ s10434-016-5544-1
2 Van Cutsem E, Sagaert X, Topal B, Haustermans K and Prenen H: Gastric cancer. Lancet 388(10060): 2654-2664, 2016. PMID: 27156933. DOI: $10.1016 / \mathrm{S} 0140-6736(16) 30354-3$

3 Razzak M: Genetics: New molecular classification of gastric adenocarcinoma proposed by the cancer genome atlas. Nat Rev Clin Oncol 11(9): 499, 2014. PMID: 25113841. DOI: 10.1038/ nrclinonc.2014.138

4 Sawaki K, Kanda M and Kodera Y: Review of recent efforts to discover biomarkers for early detection, monitoring, prognosis, and prediction of treatment responses of patients with gastric cancer. Expert Rev Gastroenterol Hepatol 12(7): 657-670, 2018. PMID: 29902383. DOI: 10.1080/17474124.2018.1489233

5 Resende C, Thiel A, Machado JC and Ristimaki A: Gastric cancer: Basic aspects. Helicobacter 16: 38-44, 2011. PMID: 21896084. DOI: $10.1111 / \mathrm{j} .1523-5378.2011 .00879 . x$

6 Lin LL, Huang HC and Juan HF: Discovery of biomarkers for gastric cancer: A proteomics approach. J Proteomics 75(11): 30813097, 2012. PMID: 22498886. DOI: 10.1016/j.jprot.2012.03.046

7 Kanda M, Nomoto S, Oya H, Takami H, Shimizu D, Hibino S, Hashimoto R, Kobayashi D, Tanaka C, Yamada S, Fujii T, Nakayama G, Sugimoto H, Koike M, Fujiwara M and Kodera Y: The expression of melanoma-associated antigen $\mathrm{d} 2$ both in surgically resected and serum samples serves as clinically relevant biomarker of gastric cancer progression. Ann Surg Oncol 23: 214221, 2016. PMID: 25743330. DOI: 10.1245/s10434-015-4457-8

8 Shimizu D, Kanda M and Kodera Y: Emerging evidence of the molecular landscape specific for hematogenous metastasis from gastric cancer. World J Gastrointest Oncol 10(6): 124-136, 2018. PMID: 29988904. DOI: 10.4251/wjgo.v10.i6.124

9 Goonetilleke KS and Siriwardena AK: Systematic review of carbohydrate antigen (CA 19-9) as a biochemical marker in the diagnosis of pancreatic cancer. Eur J Surg Oncol 33(3): 266-270, 2007. PMID: 17097848. DOI: 10.1016/j.ejso.2006.10.004

$10 \mathrm{He}$ CZ, Zhang KH, Li Q, Liu XH, Hong Y and Lv NH: Combined use of AFP, CEA, CA125 and CA19-9 improves the sensitivity for the diagnosis of gastric cancer. BMC Gastroenterol 13: 87, 2013. PMID: 23672279. DOI: 10.1186/ 1471-230x-13-87

11 Suryo Rahmanto Y, Bal S, Loh KH, Yu Y and Richardson DR: Melanotransferrin: Search for a function. Biochim Biophys Acta 1820(3): 237-243, 2012. PMID: 21933697. DOI: 10.1016/ j.bbagen.2011.09.003

12 Kanda M, Tanaka H, Shimizu D, Miwa T, Umeda S, Tanaka C, Kobayashi D, Hattori N, Suenaga M, Hayashi M, Iwata N, Yamada S, Fujiwara M and Kodera Y: SYT7 acts as a driver of hepatic metastasis formation of gastric cancer cells. Oncogene 37(39): 5355-5366, 2018. PMID: 29858600. DOI: 10.1038/ s41388-018-0335-8

13 Kanda M, Murotani K, Kobayashi D, Tanaka C, Yamada S, Fujii T, Nakayama G, Sugimoto H, Koike M, Fujiwara M and Kodera Y: Postoperative adjuvant chemotherapy with S-1 alters recurrence patterns and prognostic factors among patients with stage II/III gastric cancer: A propensity score matching analysis. Surgery 158(6): 1573-1580, 2015. PMID: 26120068. DOI: 10.1016/j.surg.2015.05.017

14 Szasz AM, Lanczky A, Nagy A, Forster S, Hark K, Green JE, Boussioutas A, Busuttil R, Szabo A and Gyorffy B: Crossvalidation of survival associated biomarkers in gastric cancer using transcriptomic data of 1,065 patients. Oncotarget 7(31): 4932249333, 2016. PMID: 27384994. DOI: 10.18632/oncotarget.10337 
15 Sakuramoto S, Sasako M, Yamaguchi T, Kinoshita T, Fujii M, Nashimoto A, Furukawa H, Nakajima T, Ohashi Y, Imamura H, Higashino M, Yamamura Y, Kurita A and Arai K: Adjuvant chemotherapy for gastric cancer with s-1, an oral fluoropyrimidine. N Engl J Med 357(18): 1810-1820, 2007. PMID: 17978289. DOI: 10.1056/NEJMoa072252

16 Sawaki K, Kanda M, Miwa T, Umeda S, Tanaka H, Tanaka C, Kobayashi D, Suenaga M, Hattori N, Hayashi M, Yamada S, Nakayama G, Fujiwara $M$ and Kodera Y: Troponin i2 as a specific biomarker for prediction of peritoneal metastasis in gastric cancer. Ann Surg Oncol 25(7): 2083-2090, 2018. PMID: 29663169. DOI: 10.1245/s10434-018-6480-z

17 Kanda M, Shimizu D, Tanaka H, Tanaka C, Kobayashi D, Hayashi M, Iwata N, Niwa Y, Yamada S, Fujii T, Sugimoto H, Murotani K, Fujiwara M and Kodera Y: Significance of SYT8 for the detection, prediction, and treatment of peritoneal metastasis from gastric cancer. Ann Surg 267(3): 495-503, 2018 PMID: 28026832. DOI: 10.1097/SLA.0000000000002096

18 Uno Y, Kanda M, Miwa T, Umeda S, Tanaka H, Tanaka C, Kobayashi D, Suenaga M, Hattori N, Hayashi M, Yamada S, Nakayama G, Fujiwara M and Kodera Y: Increased expression of DNAJC12 is associated with aggressive phenotype of gastric cancer. Ann Surg Oncol 26(3): 836-844, 2019. PMID: 30617870. DOI: $10.1245 / \mathrm{s} 10434-018-07149-\mathrm{y}$

19 Shimizu D, Kanda M, Tanaka H, Kobayashi D, Tanaka C, Hayashi M, Iwata N, Niwa Y, Takami H, Yamada S, Fujii T, Nakayama G, Fujiwara M and Kodera Y: GPR155 serves as a predictive biomarker for hematogenous metastasis in patients with gastric cancer. Sci Rep 7: 42089, 2017. PMID: 28165032. DOI: $10.1038 /$ srep42089

20 Tanaka H, Kanda M, Miwa T, Tanaka C, Kobayashi D, Umeda S, Shibata M, Suenaga M, Hattori N, Hayashi M, Iwata N, Yamada S, Nakayama G, Fujiwara M and Kodera Y: Patternspecific transcriptomics identifies ASGR2 as a predictor of hematogenous recurrence of gastric cancer. Mol Cancer Res 16(9): 1420-1429, 2018. PMID: 29784667. DOI: 10.1158/15417786.Mcr-17-0467

21 Kanda M, Tanaka C, Kobayashi D, Tanaka H, Shimizu D, Shibata M, Takami H, Hayashi M, Iwata N, Niwa Y, Yamada S, Fujii T, Nakayama G, Fujiwara M and Kodera Y: Epigenetic suppression of the immunoregulator mzb1 is associated with the malignant phenotype of gastric cancer. Int J Cancer 139(10): 2290-2298, 2016. PMID: 27459504. DOI: 10.1002/ijc.30286

22 Kanda M, Nomoto S, Oya H, Takami H, Shimizu D, Hibino S, Hashimoto R, Kobayashi D, Tanaka C, Yamada S, Fujii T, Nakayama G, Sugimoto H, Koike M, Fujiwara M and Kodera Y: The expression of melanoma-associated antigen D2 both in surgically resected and serum samples serves as clinically relevant biomarker of gastric cancer progression. Ann Surg Oncol 23: 214212, 2016. PMID: 25743330. DOI: 10.1245/s10434-015-4457-8

23 Dunn LL, Sekyere EO, Suryo Rahmanto Y and Richardson DR: The function of melanotransferrin: A role in melanoma cell proliferation and tumorigenesis. Carcinogenesis 27(11): 21572169, 2006. PMID: 16704991. DOI: 10.1093/carcin/bg1045

24 Sekyere EO, Dunn LL, Suryo Rahmanto Y and Richardson DR: Role of melanotransferrin in iron metabolism: Studies using targeted gene disruption in vivo. Blood 107(7): 2599-2601, 2006. PMID: 16291590. DOI: 10.1182/blood-2005-10-4174

25 Bertrand Y, Demeule M, Michaud-Levesque J and Beliveau R: Melanotransferrin induces human melanoma SK-Mel-28 cell invasion in vivo. Biochem Biophys Res Commun 353(2): 418423, 2007. PMID: 17196552. DOI: 10.1016/j.bbrc.2006.12.034

26 Rolland Y, Demeule M, Fenart L and Beliveau R: Inhibition of melanoma brain metastasis by targeting melanotransferrin at the cell surface. Pigment Cell Melanoma Res 22(1): 86-98, 2009. PMID: 19017294. DOI: 10.1111/j.1755-148X.2008.00525.x

27 Shin J, Kim HJ, Kim G, Song M, Woo SJ, Lee ST, Kim H and Lee C: Discovery of melanotransferrin as a serological marker of colorectal cancer by secretome analysis and quantitative proteomics. J Proteome Res 13(11): 4919-4931, 2014. PMID: 25216327. DOI: $10.1021 /$ pr500790f

28 Dus-Szachniewicz K, Ostasiewicz P, Wozniak M, Kolodziej P, Wisniewski JR and Ziolkowski P: Pattern of melanotransferrin expression in human colorectal tissues: An immunohistochemical study on potential clinical application. Anticancer Res 35(12): 6551-6561, 2015. PMID: 26637869.

29 Katoh M and Katoh M: Identification and characterization of human SNAIL13 (SNAI3) gene in silico. Int J Mol Med 11(3): 383-388, 2003. PMID: 12579345.

30 Chen C, Aihemaiti M, Zhang X, Qu H, Jiao J, Sun Q and Yu W: FOXD4 induces tumor progression in colorectal cancer by regulation of the SNAI3/CDH1 axis. Cancer Biol Ther 19(11): 1065-1071, 2018. PMID: 30252597. DOI: 10.1080/15384 047.2018.1480291

31 Honisch S, Yu W, Liu G, Alesutan I, Towhid ST, Tsapara A, Schleicher S, Handgretinger R, Stournaras C and Lang F: Chorein addiction in VPS13A overexpressing rhabdomyosarcoma cells. Oncotarget 6(12): 10309-10319, 2015. PMID: 25871399. DOI: $10.18632 /$ oncotarget.3582

32 Morisaki T, Yashiro M, Kakehashi A, Inagaki A, Kinoshita H, Fukuoka T, Kasashima H, Masuda G, Sakurai K, Kubo N, Muguruma K, Ohira M, Wanibuchi $\mathrm{H}$ and Hirakawa $\mathrm{K}$ : Comparative proteomics analysis of gastric cancer stem cells. PLoS One 9(11): e110736, 2014. PMID: 25379943. DOI: 10.1371/journal.pone.0110736

33 Jaiswal BS, Kljavin NM, Stawiski EW, Chan E, Parikh C, Durinck S, Chaudhuri S, Pujara K, Guillory J, Edgar KA, Janakiraman V, Scholz RP, Bowman KK, Lorenzo M, Li H, Wu J, Yuan W, Peters BA, Kan Z, Stinson J, Mak M, Modrusan Z, Eigenbrot C, Firestein R, Stern HM, Rajalingam K, Schaefer G, Merchant MA, Sliwkowski MX, de Sauvage FJ and Seshagiri S: Oncogenic ERBB3 mutations in human cancers. Cancer Cell 23(5): 603-617, 2013. PMID: 23680147. DOI: 10.1016/j.ccr.2013.04.012

34 Wang Z: ErbB receptors and cancer. Methods Mol Biol 1652: 335, 2017. PMID: 28791631. DOI: 10.1007/978-1-4939-7219-7_1

35 Moghbeli M, Makhdoumi Y, Soltani Delgosha M, Aarabi A, Dadkhah E, Memar B, Abdollahi A and Abbaszadegan MR: ErbB1 and ErbB3 co-over expression as a prognostic factor in gastric cancer. Biol Res 52(1): 2, 2019. PMID: 30621788. DOI: 10.1186/s40659-018-0208-1

36 Duraes C, Almeida GM, Seruca R, Oliveira C and Carneiro F: Biomarkers for gastric cancer: Prognostic, predictive or targets of therapy? Virchows Arch 464(3): 367-378, 2014. PMID: 24487788. DOI: $10.1007 / \mathrm{s} 00428-013-1533-y$

Received October 9, 2019

Revised October 21, 2019

Accepted October 22, 2019 